\title{
EVALUACIÓN DE LA SOSTENIBILIDAD DE LAS EMPRESAS FORESTALES DEL VALLE DEL MANTARO-2008*
}

\author{
Hugo Miguel Miguel ${ }^{1}$, Jesús Antonio Hurtado Rivera²
}

\begin{abstract}
RESUMEN
Se estudió la sostenibilidad de las empresas forestales más representativas del Valle del Mantaro que transforman primariamente la madera de Eucalyptus globulus Labill. Se evaluaron mediante encuestas, indicadores de gestión de las dimensiones: ambiental, social y económico de dos aserraderos, un aserradero de sierra circular y un aserradero de sierra banda. Los objetivos del estudio fueron: determinar cuantitativamente los grados o niveles de sostenibilidad de las empresas forestales del Valle del Mantaro, y analizar comparativamente la sostenibilidad ambiental, económica y social de las empresas forestales. Se concluye que las industrias forestales del Valle del Mantaro son insostenibles; asimismo, la dimensión ambiental es la más insostenible, seguidas por las dimensiones social y económica.
\end{abstract}

Palabras clave: Sostenibilidad, aserraderos, eucalipto.

\section{Evaluation of the sustainable development of the forest companies of the Mantaro Valley -2008}

\begin{abstract}
The sustainable development of the most representative forest companies in the Mantaro Valley which primarily transform the wood of Eucalyptus globulus Labill was studied. They were evaluated by means of surveys, management indicators of the: environmental, social and economic dimensions of two sawmills; a circular and a band sawmill. The objectives of the study were: to determine the grades or levels of sustainable development of the forest companies of the Mantaro Valley quantitatively, and analyze the environmental, economic and social sustainable development of the forest companies comparatively. It was concluded that the forest industries of the Mantaro Valley are untenable; also, the environmental dimension is the most untenable, continued by the social and economic dimensions
\end{abstract}

Key words: Sustainable development, sawmills, eucalyptus.

\footnotetext{
* Este trabajo de investigación fue recibido el 10/12/2008 retornado para su revisión 10/03/2009 y aprobado para su publicación 16/10/2009

1Email: hmiguel21@gmail.com

${ }^{2}$ Email: jahr38@hotmail.com
} 


\section{INTRODUCCION}

Una gran cantidad de factores influyen en el aserrado de la madera. Las variables más significativas son: el ancho de corte, el esquema de corte, las dimensiones de la madera, el diámetro, longitud, conicidad y calidad de la troza, sobredimensión de la madera verde, la toma de decisiones del personal y las condiciones de manutención de la maquinaria, (9).

La implementación de estrategias de sostenibilidad en una empresa forestal, se inicia con la conceptualización del sistema de desarrollo sostenible, etapa en la cual se realiza un análisis interno y externo de la organización para determinar su perfil de sostenibilidad, así como sus opciones de mejora en las dimensiones ambiental, económica y social, (6).
En el valle del Mantaro, las empresas forestales más representativas vienen a ser los aserraderos que transforman primariamente la madera de Eucalyptus globuIus Labill con más de 70 años de antigüedad; sin embargo, en estos últimos años se nota claramente signos de crisis, relacionado con la materia prima, la tecnología y los recursos humanos.

Para estimar la sostenibilidad de estas empresas forestales, se evaluaron mediante encuestas, los indicadores de gestión de las dimensiones: ambiental, social y económico de 2 aserraderos del Valle del Mantaro que transforman primariamente la madera de eucalipto, un aserradero de sierra circular y un aserradero de sierra banda, cuyos objetivos del estudio fueron: determinar cuantitativamente los grados o niveles de sostenibilidad de las empresas forestales del Valle del Mantaro, y analizar comparativamente la sostenibilidad ambiental, económica y social de las empresas forestales.

\section{MATERIAL Y METODOS}

La investigación se llevó a cabo en un aserradero de sierra banda y un aserradero de sierra circular, del Valle del Mantaro.

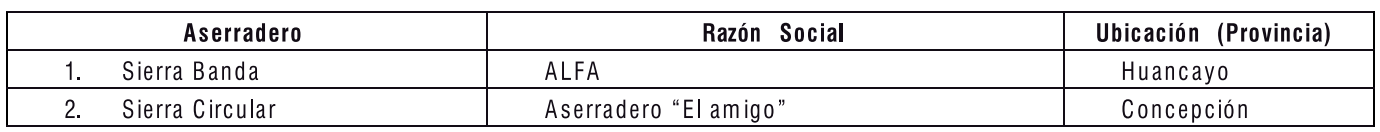

Se utilizó el Software de Autodiagnóstico de Sostenibilidad de SAFE (Sustainability Assessment For Enterprises), desarrollada por Baedeker, C., Heder, P., Klemisch, H., Rohn, H. (2002), del Instituto Wupperta, bajo el marco de una Hoja Electrónica.

Para el diseño de las encuestas se consideraron los siguientes rubros:

\section{A. Economía}

A1. Satisfacción de los requerimientos de clientes y mercados.

A2. Relación económica con los empleados.

A3. Relación económica con proveedores.

A4. Satisfacción de los requerimientos de los inversionistas.

A5. Relación económica con la sociedad.

\section{B. Medio ambiente}

B1. Organización de la gestión ambiental.

B2. Protección del medio ambiente a nivel de la producción.

B3. Desarrollo de productos tomando en cuenta el medio ambiente.

\section{Aspectos sociales}

C1. Empleo y mecanismos de evaluación.

C2. Protección de la salud y condiciones laborales.

C3. Capacitación, diversidad e incentivos.

C4. Transparencia e interacción. 


\section{RESULTADOS}

\section{Diagnostico de la sostenibilidad de un aserradero promedio de sierra banda}

La evaluación de los indicadores de las dimensiones: ambiental, social y económica del desarrollo sostenible, se puede sintetizar en forma sistémica con aplicación del software del tipo hoja de cálculo; en el siguiente gráfico, comúnmente conocido como "telaraña", muestra en forma de conjunto el grado de desarrollo sostenible, como también de manera lineal, o mejor dicho de acuerdo a las tres dimensiones del desarrollo sostenible; asimismo, la subsiguiente tabla muestra los valores numéricos de la evaluación de acuerdo a las dimensiones y en las formas especificas, dentro de cada dimensión; es este caso de un aserradero promedio del Valle del Mantaro de sierra de banda, que tecnológicamente es muy diferente que los aserraderos de sierra circular.

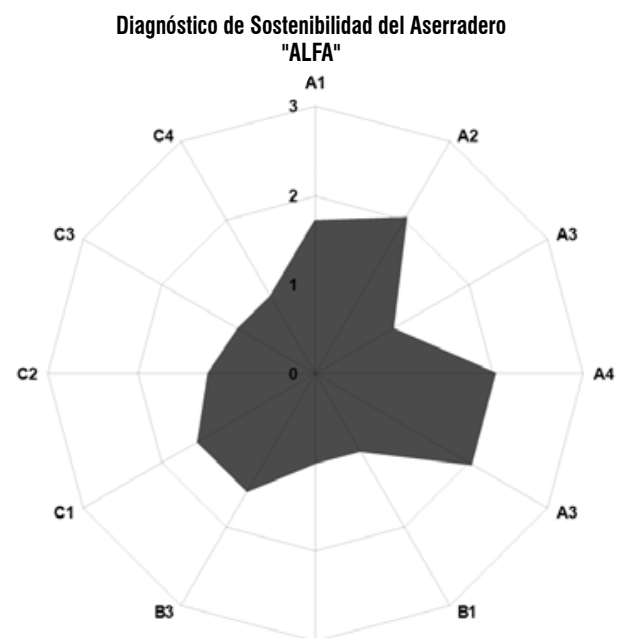

\begin{tabular}{|c|c|}
\hline \multicolumn{2}{|c|}{ Aserradero ALFA } \\
\hline A1 & 1.714285714 \\
\hline A2 & 2 \\
\hline A3 & 1 \\
\hline A4 & 2 \\
\hline A3 & 2 \\
\hline B1 & 1 \\
\hline B2 & 1 \\
\hline B3 & 1.5 \\
\hline C1 & 1.5 \\
\hline C2 & 1.2 \\
\hline C3 & 1 \\
\hline C4 & 1 \\
\hline
\end{tabular}




\section{Diagnostico de la sostenibilidad de un aserradero promedio de sierra de circular}

De igual forma que en el caso anterior, se muestra en forma de conjunto el grado de desarrollo sostenible, como también de manera lineal, o mejor dicho de acuerdo a las tres dimensiones del desarrollo sostenible de un aserradero promedio de sierra circular, muy común en el Valle del Mantaro; asimismo, la subsiguiente tabla muestra los valores numéricos de la evaluación de acuerdo a las dimensiones y en las formas especificas, dentro de cada dimensión.

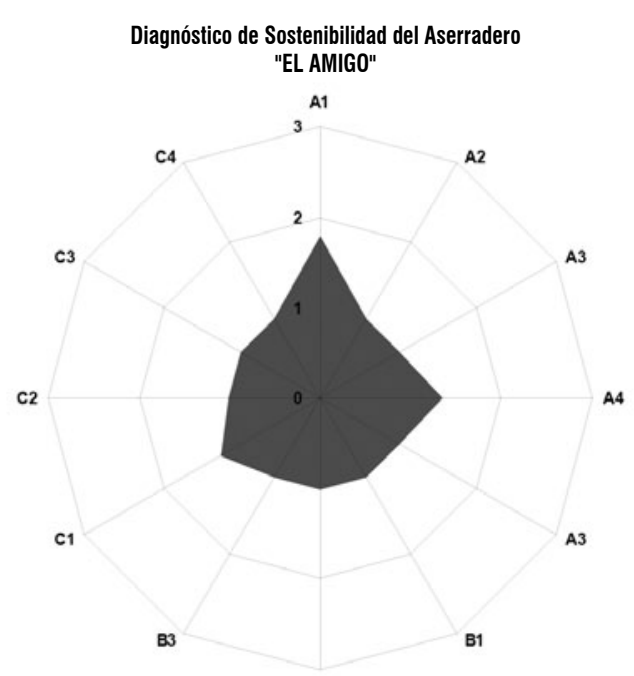

\begin{tabular}{|l|c|}
\hline \multicolumn{2}{|c|}{ Promedios de la evaluación } \\
Aserradero "EL AMIG0"
\end{tabular}

\section{DISCUSION}

Los biogramas son programas informáticos que sirven para medir el grado de desarrollo sostenible de un área geográfica determinada, (1), en el caso específico de los aserraderos del Valle del Mantaro, también es posible medir el grado de sostenibilidad integralmente las dimensiones: económica, social y ambiental, utilizando este tipo de software, mediante encuestas realistas de los desempeños de las empresas privadas.

El aserradero ALFA, es considerado como una de las empresas más sobresalientes en el rubro de la transformación primaria de la madera de eucalipto en el Valle del Mantaro; se le ha sometido una encuesta de 62 preguntas, distribuidas en las dimensiones: económica, dentro de esta los temas: satisfacción de los requerimientos de clientes y mercados, relación económica con los empleados, relación económica con proveedores, satisfacción de los requerimientos de los inversionistas y la relación económica con la sociedad; dentro de la dimensión ambiental, los temas: organización de

la gestión ambiental, protección del medio ambiente a nivel de la producción y el desarrollo de productos tomando en cuenta el medio ambiente; y dentro de la dimensión social, los temas: empleo y mecanismos de evaluación, protección de la salud y condiciones laborales, capacitación, diversidad e incentivos, y transparencia e interacción.

La dimensión económica del sistema del Aserradero ALFA, tiene un nivel de sostenibilidad relativamente aceptable, probablemente por efecto de los precios de los metales relativamente altos, por lo que el gran porcentaje de la producción de esta empresa forestal es absorbida por la actividad minera. La dimensión ambiental es la más insostenible, por efecto de la indeferencia de los propietarios de los aserraderos de los temas ambientales y de la escasez de los árboles de eucalipto apto para el proceso de aserrío; de la misma manera, la dimensión social también es insostenible porque al igual que en el caso de la dimensión ambiental, los propietarios no muestran responsabilidad social frente al bienestar de los trabajadores forestales. En términos 
generales, se podría afirmar que el sistema del aserradero promedio de sierra cinta del Valle del Mantaro, se encuentra en un estado de insostenibilidad severa, mostrando debilidades muy marcadas en las tres dimensiones de la sostenibilidad, tal como puede observarse en el primer gráfico, (10).

Por otro lado, la evaluación del grado de sostenibilidad en el aserradero "El Amigo", también arrojó un alto grado de insostenibilidad, mucho más significativo que en el caso del aserradero ALFA, debido a las mismas causas que se explicaron en el caso de este aserradero; en efecto, la interpretación de estos indicadores de sostenibilidad nos alertan que la industria forestal del Valle del Mantaro está en un proceso de insostenibilidad que podría significar a corto plazo el colapso de estas industrias; no existen políticas de desarrollo forestal claros, todo lo contrario, las instituciones del sector y los "industriales" tan solamente piensan en sus beneficios personalizados, sin criterios prospectivos, (11).

\section{LITERATURA CITADA}

- Sepúlveda. S. Metodología para estimar el nivel de desarrollo sostenible en territorios rurales. Costa Rica; 2005.

- Nieto LM. ¿Cómo sabemos si tenemos avances hacia el desarrollo sostenible? San Luís Potosí, México; 2002.

- Castro M. Indicadores de desarrollo sostenible urbano. Una aplicación para Andalucía. Tesis Doctoral. Universidad de Málaga; 2002.

- Velásquez L. Propuesta de una metodología de planificación para el desarrollo urbano sostenible y diseño de un sistema de evaluación de la sostenibilidad de ciudades medianas de América Latina. Tesis Doctoral. Universidad Politécnica de Catalunya; 2003.

- Funge C. Ciudades europeas sostenibles. Informe. Comisión Europea. Bruselas; 1996.

- Alimentos Campestres S.A. Reducción de la pobreza y mejoramiento del ambiente. Una estrategia de desarrollo integral sostenible. Guatemala; 2006.

- Comisión sobre Desarrollo Sostenible. ONU. Indicadores de desarrollo sostenible marco y metodologías. Santiago de Chile; 2001.
- Sepúlveda S, et al. Metodología para estimar el nivel de desarrollo sostenible en espacios territoriales. Costa Rica; 2002.

- Spicher OA. Aprovechamiento en el aserrado de seguoia (Sequoia sempervirens (D.Don) Ende.) y clasificación de la madera obtenida. Tesis Ing. de la Madera. Facultad de Ciencias Forestales, Universidad de Chile; 2004-

- Nasca FA, Toranzos M, Banegas NR. Evaluación de la sostenibilidad de dos modelos ganaderos de la llanura deprimida salina de Tucumán, Argentina. Zootecnia Trop., 24 (2):121-6; 2006.

- Castillo E. Evaluación de los niveles de desarrollo sostenible en espacios territoriales (granjas de producción sostenible) en provincias centrales. Investig. Pens. Crit. Panamá (2004) 2:10-8.

- Miranda T, Machado H, Suset A, Campo M, Duquesne P, Cruz A. Evaluación de sostenibilidad. Estudio de caso en una unidad básica de producción cooperativa. Estación Experimental de Pastos y Forrajes "Indio Hatuey", Matanzas, Cuba; 2006. 